\title{
Relationship between Menstrual Cycle and Effect of Relaxation Resulting from a Breathing Exercise
}

\author{
Motoko Ohira ${ }^{1}$, Junko Muramoto ${ }^{1}$, Shin Saito ${ }^{1}$ \\ ${ }^{1}$ Mie Prefectural College of Nursing \\ motoko.ohira@mcn.ac.jp
}

\begin{abstract}
The aim of this study examine the difference caused by relaxation resulting from a breathing exercise in healthy females during menstrual cycle as measured by power spectral analysis of heart rate variability (HRV). The subjects were 15 women, aged 21- 39 years. The effect of the breathing exercise was measured in terms of HRV before and after a mental task, in both follicular and luteal phases. The results showed that the high-frequency (HF) was increased significantly by the breathing exercise, and the LF/HF ratio was not changed significantly in follicular phase. These results indicated that the parasympathetic nervous system activity was increased. On the other hand, HF was not changed significantly by the breathing exercise in luteal phase. Moreover, the LF/HF ratio increased significantly. These results indicated that tension of the sympathetic nervous system was continuous. Therefore, the effect of the relaxation was exhibited in follicular phase, and not in luteal phases. It will be necessary to consider other effective methods including continuous breathing exercise for the relaxation in luteal phase.
\end{abstract}

Keywords: relaxation, breathing exercise, menstrual cycle, heart rate variability, power spectral analysis

\section{Introduction}

The life of the modern woman is busy with work and raising children and is often stressful, increasing need for rest. Adequate rest and relaxation is important for maintaining and improving women's health. However, due to the menstrual cycle, women experience changes in mood, physical condition, and susceptibility to fatigue. This study used heart rate variability to investigate the menstrual cycle and the effectiveness of breathing exercises in attaining relaxation, with a focus on a breathing exercise that can be easily incorporated into daily life.

\section{Methods}

\section{2-1. Subjects}

The subjects were 15 women between 21 and 39 years of age who consented to participate in this study. The subjects were taking no drugs, including oral contraceptives, and were non-smokers. The subjects were prohibited from eating within $2 \mathrm{hr}$ before the experiment and from ingesting caffeine on the day of the experiment. They were asked to sleep adequately the day before the experiment.

The subjects were randomized to a group that was tested during the follicular phase ( 8 subjects) and a group that was 
tested during the luteal phase ( 7 subjects). Basal body temperature was measured and recorded for all of the subjects.

\section{2-2. Breathing exercise}

The breathing exercise involved closing the eyes and decreasing the respiration rate to produce a pattern of slow breathing, with exhalation lengthened relative to inhalation. Subjects were instructed to release tension in the body and relax during exhalation.

\section{2-3. Procedure}

Experiment was performed under 2 conditions, once without (control experiment) and once with the use of the breathing exercise (breathing experiment). All subjects participated in both experiments. The control and breathing experiments were performed on different days for all subjects. The experiment was performed with the subjects sitting in a chair in a room at $25^{\circ} \mathrm{C}$ and $45 \%$ humidity.

The experimental procedure is shown in Fig. 1. In preparation for the experiment, electrocardiogram (ECG) electrodes and a respiratory thermistor sensor were attached to the subjects. The subjects were then asked to sit at rest with their eyes open for 10 minutes. After the preparations were complete, experiment was started. The subjects rested with their eyes closed for 5 minutes (prerest), then performed single-digit addition tasks for $15 \mathrm{~min}$ using a personal computer. Subsequently, the subjects rested for $10 \mathrm{~min}$ with their eyes open for the control experiment or implemented the prescribed breathing exercise for $10 \mathrm{~min}$ for the breathing experiment. At the end of the procedure, the subjects rested for $5 \mathrm{~min}$ with their eyes closed (post-rest). During the pre- and post-rests, a metronome was used to control respiration rate so that the subjects paced breathing at 15 breaths/minute.

\section{2-4. ECG and Respiration analysis}

\section{2-4-1. Heart rate variability}

ECG measurements were performed using a BA-1008 bioamplifier (Teac Instruments) and $\mathrm{CM}_{5}$ leads. The ECG waveforms were input into a computer at a sampling frequency of $1 \mathrm{KHz}$, and the RR interval was extracted and cubic spline interpolation performed. For supplementary data, $2 \mathrm{~Hz}$ resampling and Hanning window processing were performed. The use of the fast Fourier transform in power spectrum analysis was then performed based on data obtained for 5 continuous minutes. The analysis software used was Analog Recorder Pro, version 1.60 for Windows (G1 Systems). In the analysis,

\begin{tabular}{|c|c|c|c|c|c|}
\hline \multicolumn{2}{|c|}{ Preparation } & \multicolumn{4}{|c|}{ Experiment } \\
\hline \multirow[t]{4}{*}{ Preparation } & \multirow{2}{*}{$\begin{array}{c}\text { Rest with } \\
\text { their eyes } \\
\text { open } \\
10 \text { minutes }\end{array}$} & \multirow{2}{*}{$\begin{array}{l}\text { Rest with } \\
\text { their eyes } \\
\text { closed } \\
\text { (pre-rest) }\end{array}$} & \multirow{2}{*}{$\begin{array}{l}\text { Single-digit addition } \\
\text { tasks using a personal } \\
\text { computer }\end{array}$} & $\begin{array}{c}\text { Control experiment } \\
\text { (Rest with their eyes } \\
\text { open) }\end{array}$ & \multirow[t]{2}{*}{$\begin{array}{c}\text { Rest with } \\
\text { their eyes } \\
\text { closed }\end{array}$} \\
\hline & & & & $\begin{array}{c}\text { Breathing } \\
\text { experiment. }\end{array}$ & \\
\hline & & (5 minutes) & (15 minutes) & (10 minutes) & (5 minutes) \\
\hline & & 0 & & & \\
\hline
\end{tabular}

Fig. 1 Experimental procedure 
0.04 to $0.15 \mathrm{~Hz}$ was considered low frequency (LF) and 0.15 to $0.40 \mathrm{~Hz}$ was considered high frequency (HF).

\section{2-4-2. Respiration}

To control the respiration rate, the rate was measured using respiratory thermistor sensors attached to the subject's nose and mouth.

\section{2-5. Statistical analysis}

The pre- and post-rest control and breathing experiments were compared for the follicular and luteal phases. The Wilcoxon test was used for statistical analysis.

\section{Results and Discussion}

The at-rest respiration rate in the follicular and luteal phases was steady at approximately $0.25 \mathrm{~Hz}$ (13-15 breaths/min).

The change in HF between pre- and post- rest in the follicular phase is shown in Fig. 2. In the control experiment, no significant differences were seen between the pre- and post- rest periods. However, in the breathing experiment, a significant difference was seen between the pre- and post- rest periods, with the value being significantly higher for the post- rest $(p<0.05)$.

The change in the LF/HF ratio (LF/HF) between the preand the post- rest periods in the follicular phase are shown in Fig. 3. No significant difference was seen between the pre- and post- rest periods in the control and breathing experiments. That is, in the follicular phase, a significant increase was seen in HF, an index of parasympathetic nervous system activity, while no significant difference was seen in LF/HF, an index of sympathovagal balance.

The change in HF between the pre- and post- rest periods in the luteal phase is shown in Fig. 4. No significant difference was seen between the pre- and postrest periods in the control and breathing experiments.

The change in LF/HF between the pre- and post- rest periods in the luteal phase is shown in Fig. 5 . In the control experiment, no significant difference was seen between the pre- and post- rest periods. However, a significant difference was seen between the pre- and postrest periods in the breathing experiment, with the value being significantly higher for post- rest $(\mathrm{p}<0.05)$. Thus, in the luteal phase, a significant increase was seen in LF/HF, an index of sympathovagal balance, while no significant difference was seen in $\mathrm{HF}$, an index of parasympathetic nervous system activity.

In the follicular phase, sympathetic nervous activity is increased by estrogen, a follicular hormone [1]. The effect of estrogen in this phase is known to result in a higher value for $\mathrm{HF}$ than in the luteal phase. It has been surmised that this type of physiological mechanism improves the relaxation effect of the breathing exercise during the follicular phase due to increased parasympathetic nervous activity resulting from the effect of estrogen.

During the luteal phase, on the other hand, it has been speculated that the sensitivity of the baroreceptors is decreased, post-stress heart rate recovery is delayedand sympathetic tone is high, $[2,3]$ due to the effect of the luteal hormone, progesterone. This may explain the inadequate effect of the breathing exercise during the luteal phase.

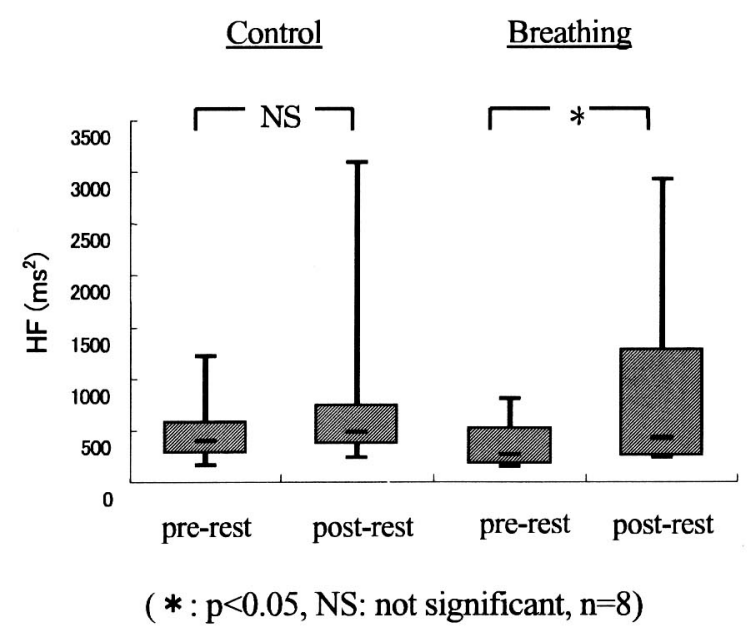

Fig.2 Changes in the high frequency (HF) during the follicular phase. 


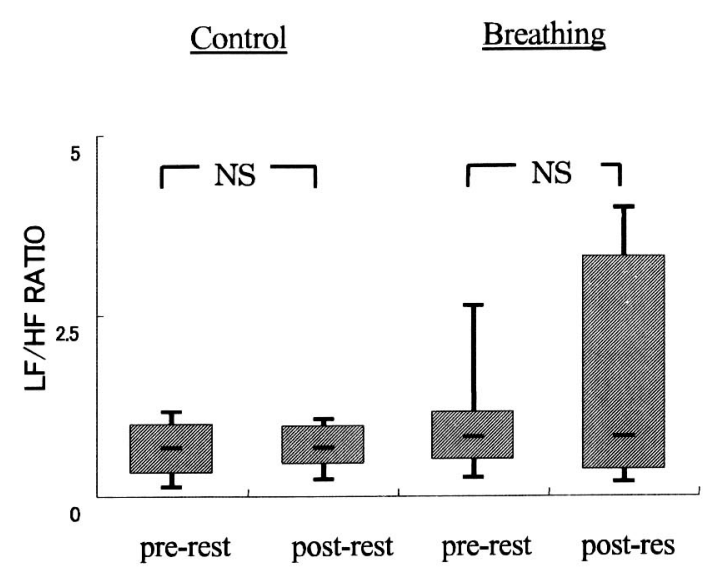

(NS: not significant, $n=8$ )

\section{Fig. 3 Changes in the LF/HF RATIO during the follicular phase.}

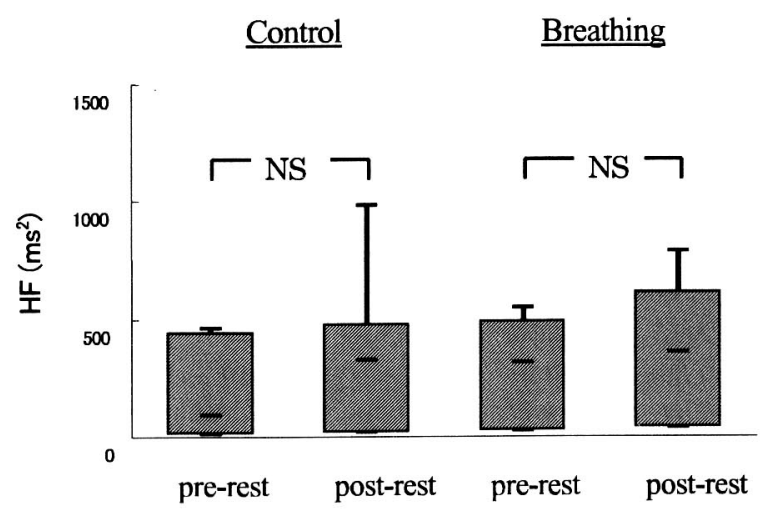

(NS: not significant, $\mathrm{n}=7$ )

\section{Fig.4 Changes in the high frequency (HF) during the luteal phase.}

\section{Conclusion}

The relaxation effect of a breathing exercise for women was evaluated based on heart rate variability. In the follicular phase, HF was significantly increased after implementation of the breathing exercise, indicating that

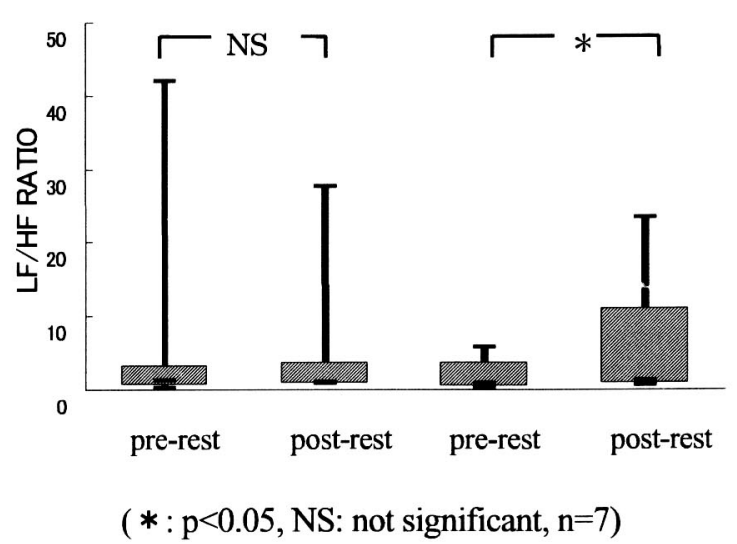

Fig.5 Changes in the LF/HF RATIO during the luteal phase.

the technique had a relaxation effect.

It will be necessary to consider other effective methods including continuous breathing exercise for the relaxation in luteal phase.

\section{References}

[1] Heikki V. Huikuri , Sirkku M.Pikkujamsa,K.E.Juhani Airaksinen: Sex-related differences in autonomic modulation of heart rate in middle-aged subjects,Circulation, 94(2),122 125,1996.

[2] Cheryl M.Heesch and Richard C.Rogers: Effects of pregnancy and progesterone metabolites on regulation of sympathetic outflow, Clinical and Experimental Pharmacology and Physiology, 22,136 142, 1995.

[3] Mariko Nakamura,Kouichirou Hayashi,Katsuji Aizawa,Fumie Murai and Noboru Mesaki: Changes in cardiac autonomic nervous activity during menstrual cycle of young women, Jpn.J.Phys.Fitness Sports Med, 51, 307 316,2002. 